\title{
The laparoscopic assisted duhamel pull through procedure for hirschsprung's disease: Our technique and short term results
}

\begin{abstract}
Aims: To prospectively evaluate the laparoscopic assisted Duhamel pull through (LADPT) procedure for Hirschsprung's disease at our centre.

Material and methods: After clinico-radiological diagnosis of Hirschsprung's disease junctional colostomy and mutltiple seromuscular biopsy was taken. After histological confirmation of diagnosis definitive surgery was done by laparoscopic assisted Duhamal procedure. Mobilization of aganglionic bawel and colon proximal to leveling colostomy was done laproscopically. The leveling colostomy taken down, bowel divided at the ganglionic segment, proximal colon pulled into retro-rectal avascular tunnel, all through the colostomy incision. Transanal stapled side to side colo-rectal anastomosis was done with rectal stump closure.
\end{abstract}

25 LADPT were done from March 2009 - 2011. This was retrospectively compared with the 25 cases of open Duhamel procedures.

Results: Mean age of surgery was $6.0 \pm 0.23$ months comparable in both groups (ranged 3 $\mathrm{m}-5 \mathrm{yrs}$ ). Mean operative time, blood loss and hospital stay with range were $80 \pm 0.30$ (70100 minutes), $50 \pm 0.20$ (40-90 ml) and 6 \pm 0.21 (3-12 days) less in LADPT cases compared with open Duhamel ( $\mathrm{p}$ value.008, 0.0067 and.009 respectively). Per-operative one left ureteric injury occurred in LADPT. In one year of follow-up revision LADPT (for neuronal intestinal dysplasia) and re-exploration due to small bowel stricture were needed in one patient each, there was one death due to fulminant enterocolitis.

Conclusions: In terms of ease of mobilization of the rectum and sigmoid colon, hemostasis, shorter operative time, less analgesic requirement, early postoperative recovery and small abdominal scar LADPT is a feasible procedure for Hirschsprung's Disease with a junctional colostomy.
Volume 7 Issue 2 - 2017

\author{
Sunita Singh,' Ashish Wakhlu, ${ }^{2}$ Intezar \\ Ahmad, ${ }^{3}$ Niraj Kumar Srivastava ${ }^{4}$ \\ 'Department of Pediatric Surgery, All India Institute of Medical \\ sciences, Raipur, India \\ ${ }^{2}$ Department of Pediatric Surgery, King George Medical \\ University, Lucknow, India \\ 3.Department of Pediatric Surgery, All India Institute of Medical \\ Sciences, India \\ ${ }^{4}$ Department of General Surgery, Raipur Institute of Medical \\ Sciences, Raipur, India
}

\begin{abstract}
Correspondence: Sunita Singh, Department of Pediatric Surgery, All India Institute of Medical sciences, Tatibandh. G.E. Road, Raipur, India, Tel 8518881733 ,
\end{abstract} Email drsunitasingh28@gmail.com

Received: February 18, 2017 | Published: September 18, 2017

\section{Laparoscopic assisted duhamel procedure}

\section{Introduction}

The Hirschsprung's disease (HD) is characterized by absence of ganglion cells in the Myentric and Meissners plexus' with nerve bundle hyperplasia. ${ }^{1,2}$ The surgery for HD is changed from multistage surgery to single stage. Further, till date the superiority of either Duhamel, Swenson or soave procedure is comparable. It is unclear if one of these techniques yields significantly better general (execution and timing of procedures) or and disease-specific outcomes. Most of the pediatric surgeons are stick to the surgical procedures of their choice. ${ }^{3}$. In the Duhamel procedure, a section of aganglionic rectum is left in situ and anastomosed to pulled down segment of ganglionic colon (side-toside) as a pouch reservoir. Duhamel procedure has gained popularity due to technical ease, minimal anal stretching and better visibility in entire procedure. ${ }^{2,3}$ The author has been performing the open staged Duhamel operations for HD since 20 yrs and laparoscopic assisted Duhamel pull through (LADPT) for last 2 years. The aim of this study was to review technique and early follow-up results of LADPT and compare it with open Duhamel procedure.

\section{Material and methods}

The observational study was done from Dec 2009 to Dec 2011. On clinico-radiological suspicion of $\mathrm{HD}$, all patients underwent primary decompressing colostomy with multiple leveling biopsy from the, collapse bawel, transition zone and colostomy site. These cases were compared with record analysis of 25 cases of two stage Open Duhamel procedure. All these patients were histopathologically confirmed recosigmoid type HD. The definitive surgery was performed once baby gained adequate weight and confirmation of ganglion cells from the colostomy site. The choice of approach (laparoscopic vs open) was individual case basis.

To make both groups comparable we excluded HD patients having acute enterocolitis, massively dilated rectum (delayed referral) and long segment (transition zone crossing the transverse colon) HD. Before the surgery, whole gut preparation for by polyethylene glycol and rectal wash with warm water was done. The patients were operated under general anesthesia with intravenous antibiotics at the induction of anesthesia. Indwelling catheterization and nasogastric intubation were done. The patients were positioned supine at the end of operating table in Trendlenberg position with the head end 20 degree lowered. If the child could be accommodate across the table, we preferred to place them transversely across the operation table. Older children were positioned in lithotomy position with legs secured on padded leg rests (Figure 1). The patient was secured to the table to prevent slipping with table tilts. The surgeon preferably stands at the head end of patient, with the cameraman right of the surgeon and second assistant left to the patient. The child was prepared below the nipple line up to toes, with coverage of lower limbs with sterilized leg stockings. Local anesthesia $(0.25 \%$ bupivacane at $1 \mathrm{ml} / \mathrm{kg}$ body 
weight) was infiltrated at the proposed port insertion site. First $5 \mathrm{~mm}$ $\mathrm{mm}$ port was placed by open technique through the umbilicus. The capnoperitoneum was created at initial flow rate $2 \mathrm{lit} / \mathrm{min}$ to maintenance rate at $5 \mathrm{lit} / \mathrm{min}$. Our maximum level for intraabdominal pressure was $6 \mathrm{~mm}$ of mercury, below six months of age; from 6 to 8 $\mathrm{mm}$ of mercury from six months to twelve year and $10 \mathrm{~mm}$ of mercury beyond twelve years. Continuous capnography was done to kept the end expiratory carbon-di-oxide concentration below 32. Inspection of the peritoneal cavity was done. The $2 \mathrm{nd}$ and $3 \mathrm{rd} 3 \mathrm{~mm}$ ports were placed under laparoscopic guidance. Usually these ports were in right and left flank, $6 \mathrm{~cm}$ away from midline and $2.5 \mathrm{~cm}$ above the transverse umbilical plain. In young infants the accessory instruments can also placed transcuaneously (without port).

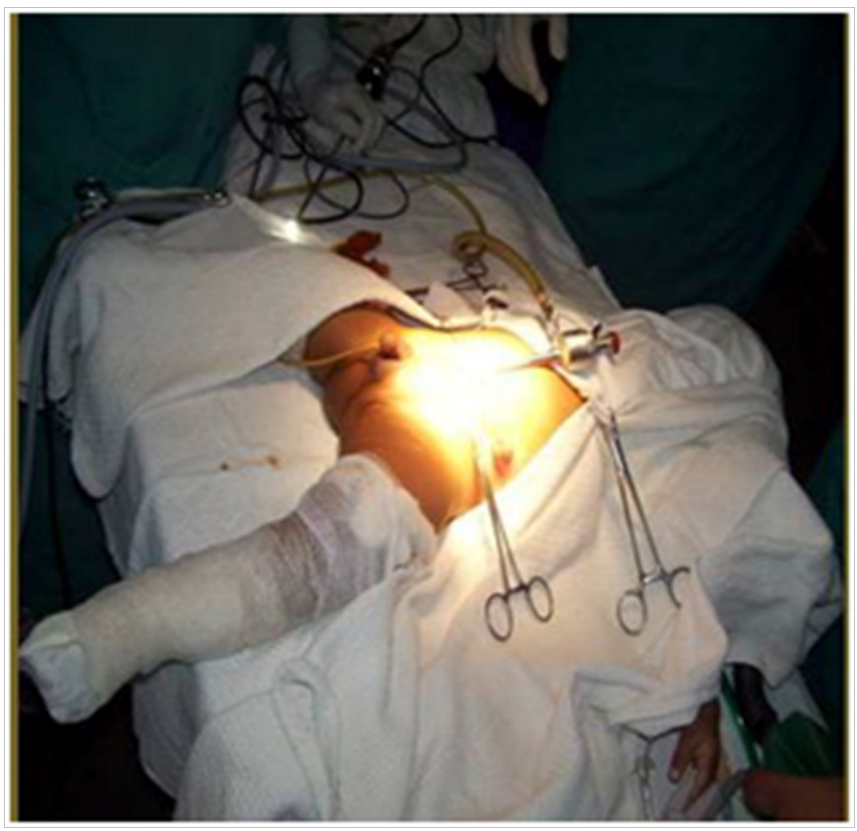

Figure I Patient positioned across the table with body draped from below the nipple.

Rectal dissection was started at the peritoneal reflection by L-shaped hook and Maryland forceps. Mesorectum was dissected by monopolar cautery, the sigmoidal vessels were coagulated by power blade (bipolar cautery) (Figure 2). Dissection in the aganglionic and transition zone was done close to the bowel wall, because this part was subsequently excised. The dissection of colon proximal to leveling colostomy was beyond the marginal artery, thus safeguarding collateral vessels. The Toldt fascia was also sectioned, if the mobilized colon was inadequate to reach up to anal verge. The smaller vessels (up to $5 \mathrm{~mm}$ in diameter) were coagulated by bipolar cautery attached to Maryland forceps, while larger vessels (up to $8 \mathrm{~mm}$ ) were controlled by dual action (coagulation followed by cutting) $5 \mathrm{~mm}$ LiNA tripol power blade vessel sealing device (Medical ApS-Formervangen, Denmark). Laparoscopically retro rectal space is created till the pelvic floor is visualized and colonic mobilization was done till ganglionic colon is reaching tension free upto pelvic floor. Under laparoscopic guidance colostomy was also dismanteled which further minimized the abdominal wound. The pneumoperitoneum was released at this point. Through colostomy incision under direct vision retrorectal tunnel was created in relatively avascular plane. The perineal surgery was started by making a curved incision in the posterior rectal wall 5 $\mathrm{mm}$ above the dentate line from 3' to 9' o clock position over the large gauze peanut placed as a guide transperitoneally in retrorectal space.
The internal sphincter and mucosa were thus divided. The colostomy site was divided, freshened (reduce the bulk of pulled colon) and its viability ensured. The colon was pulled down through posterior rectal incision without twisting. The pulled colon was anastomosed to the upper and lower lip of posterior rectal wall incision with stay sutures to hold the ends of common wall of rectum and pulled colon. Side to side recto-colon anastomosis was done by $75 \mathrm{~mm}$ proximate linear cutter GI stapler (Ethicon Endo-surgery, LLC Maxico, USA) by a single smooth uninterrupted fire. Through abdominal wound the redundant aganglionic rectum was divided proximally by $55 \mathrm{~mm}$ linear cutter GI stapler leaving behind $0.5 \mathrm{~cm}$ aganglionic rectal stump above colo rectal anastomosis (Figure 3). We thought $0.5 \mathrm{~cm}$ colorectal septum does not cause spur effect. The mesenteric window of pulled colon was closed, and the abdomen and port sites closed after lavage. We didn't placed intraperitoneal drain. Postoperatively intravenous fluids and parentral antibiotics were administered for 3 days and oral antibiotics for a further 7 days. The nasogastric tube was usually removed on the second postoperative day and oral feeding commenced.

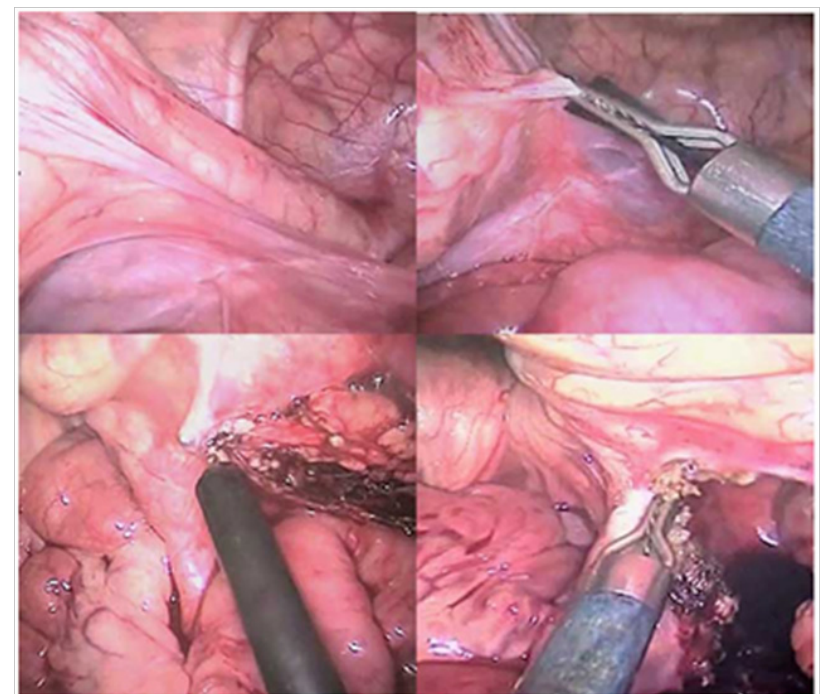

Figure 2 (A) Laparoscopic view showing spastic (aganglionic) segment and transition zone, (B) Mesocolon division by LiNA power blade, (C) Mesorectal dissection by hook cautery, (D) Major vessels sealing by bipolar dual action power blade.

\section{Results}

A total 25 patients underwent two stage LADPT presented over 2 years of the study period. These cases were compared with record analysis of 25 cases of two stage Open Duhamel procedure. The male to female ratio was $24: 1$. The mean age of operation was $6.0 \pm 0.23$ months (ranged 3 m-5 year). One child had clinical features of Down syndrome with ventricular septal defects in the heart. The mean operative time, blood loss and postoperative hospital stay by LADPT was $80 \pm 0.30$ (ranged 70-100 minutes), 50 \pm 0.20 (ranged 40-90 ml) and $6 \pm 0.21$ (ranged 3-12 days) respectively. The mean operative time, blood loss and postoperative hospital stay by open Duhamel was 120 \pm 0.50 (ranged 120-180 minutes), $90 \pm 0.10$ (ranged 100-250 ml) and $10 \pm 0.33$ (ranged 10-15 days) respectively. Thus operative time, blood loss and postoperative hospital stay was less in LADPT compared to open Duhamel ( $p$ value $0.008,0.0067$ and 0.009 respectively). Further less postoperative analgesic requirement, early enteral nutrition early mobilization in LADPT group. We have one left ureteric injury in 
LADPT due to dense adhesions with the sigmoid mesocolon. The injured ureter was repaired laparoscopically by interrupted sutures over a ureteric stent. There was no conversion to open procedure in any case.

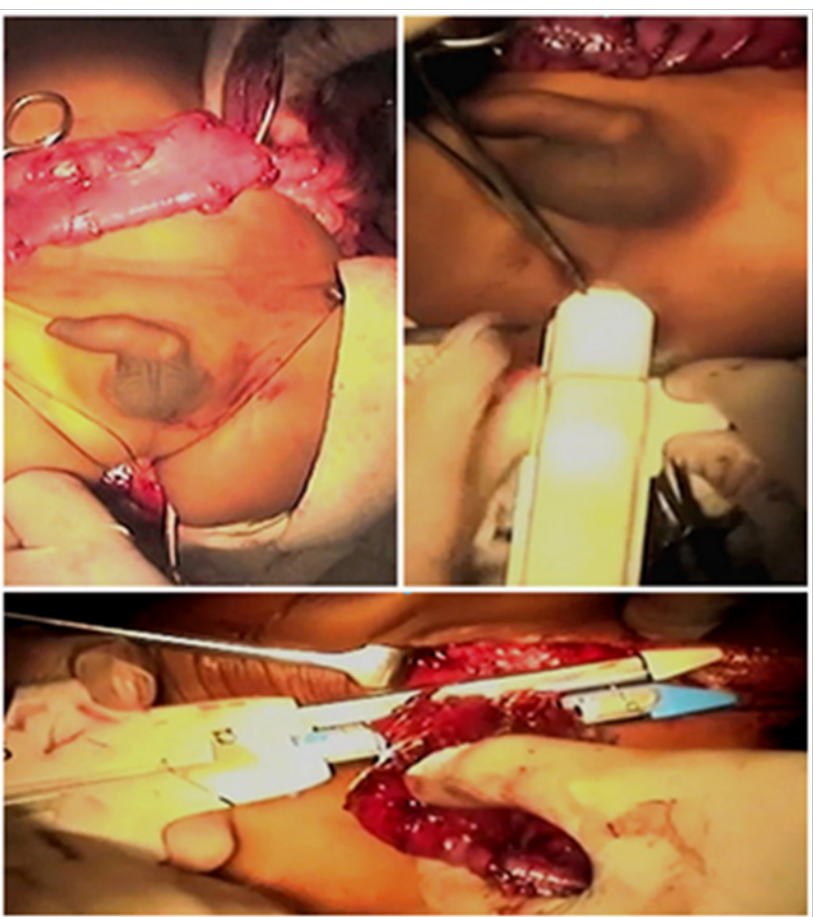

Figure 3 (A) Retrorectal pull through of ganglionic segment, (B) Trans anal side to side colo-rectal anastomosis by proximate linear cutter stapler, (C) Per abdomen division and closure of proximal aganglionic rectal stump by proximate linear cutter stapler.

In one year of follow-up one infant died after 2 month of LADPT because of repeated attack of fulminant enterocolitis. One child had persistent constipation after LADPT. The radiological work up didn't reveled twisting of bowel, anaastomosis stenosis, spur effect or bowel stricture. This child underwent revision LADPT because of suspected acquired agangliosis, but histopathology of revised bawel segment showed features of neuronal intestinal dysplasia. There was no leakage, no stenosis, no fecoloma formation or incontinence in any other patient. However, 6 of these 25 patients are below 3 years and not toilet trained, so long term results of this study are still awaited.

\section{Discussion}

The incidence of HD is 1 in 5,000 live births. ${ }^{1,2}$ The HD was first described in 1886 , since than many procedures has been described for its management. ${ }^{1-8}$ In recent years laparoscopy has evolved in the management of HD from extramucosal biopsy to primary definitive management. ${ }^{3}$ In 1994, Smith et al. reported the first successful laparoscopic Duhamel pull-through. ${ }^{8}$ In 1995, Georgeson first described the primary laparoscopic pull-through for HD. ${ }^{1}$. Three years later, De la Torre-Mondragón described the purely transanal endorectal pull-through, without the aid of laparotomy/ laparoscopy. ${ }^{9}$ Drawbacks to this procedure were the lack of seromuscular guiding biopsies, which results in removing an unnecessarily large or small segment of bowel. ${ }^{10,11}$ Further, mobilizing the rectum without dissection of the mesentery and peritoneal reflections makes this technically difficult and colonanal anastomosis under tension with absence of the natural anorectal angle. ${ }^{10}$ The Duhamel operation is best suited for a "failed" Swenson operation, long-segment HD and total colonic agangliosis.
It is also useful in cases of difficult mucosectomy viz repetitive attack of enterocolitis make the dissection difficult and grossly dilated hypertrophic rectum. ${ }^{5}$ It is important that surgeons managing patients with HD on a regular basis choose with one method in order to acquire the skill and experience necessary for consistently good results, thus at our centre we routinely perform Duhamel procedure until unless there is a specific indication for another procedure. ${ }^{11}$ Although, laparoscopic correction of long segment HD was well described in the literature, we have not tried the same in any of our patients. ${ }^{12}$

The three most popular pull-through procedures viz (Swenson's, Duhamel's and Soave's) can be performed primarily with laparoscopy with frozen section confirmation of the diagnosis and status of transition zone. However, a frozen section is not readily available at our center, thus we staged the procedure in all patients. ${ }^{4,8}$ Further, in our opinion the efficacy of staged Duhamel procedure is much better in our scenario, because most of the neonates referred late to us with low general condition, enterocolitis or colonic perforations where decompressing colostomy is life saving. ${ }^{13}$ in first stage multiple seromuscular biopsies was done via open methods and biopsy site was marked by fine nonabsorbable sutures for preoperative identification in next stage. All these procedures were done in emergency operation theatre by senior residents. Many children presenting late had features of chronic constipation and malnutrition, where colostomy was needed to improve the general health of the patients. ${ }^{14}$

Most of the pull through procedure used combination of intraluminal and linear staplers, or sole intraluminal stapler, but we have used linear cutter stapler only for the management. ${ }^{4,5,7}$ Our choice for two stage Duhamel over soave was due to the preference of the surgeon. ${ }^{3,15-17}$

The laparoscopic Duhamel has less pain, quicker recovery, and better cosmesis because it needs minimal bowel handling, thus less postoperative paralytic ileus facilitating early resumption of enteral nutrition. ${ }^{3-5}$ We also found that the LADPT has excellent exposure of peritoneal cavity, and minimal pelvic dissection preserve vital pelvic structures, peroperative third space fluid loss and decreases the risk of immediate/ long term postoperative adhesive obstruction. ${ }^{4}$ Because of excellent hemostasis achieved by vessel sealing devices during mesocolon dissection and hemostatic effect of stapler in colon transection, only five patients required perioperative blood transfusion, thus LADPT avoids the inadvertent side effects of blood transfusion also. The time required in our technique of LADPT is much lower than open Duhamel because of avoidance of making and closing a long incision, quick vessel sealing by power blade (compared to conventional suture ligation of the vessels) and quick resection and closure by staplers contrary to manual closure of rectal stump. The short operative time decreases the risk of hypothermia. Minimal requirement of postoperative analgesia allowed smooth postoperative recovery, early mobilization and short hospital stay. The avoidance of long abdominal incision not only cosmetically better but decreases the risk of scar site bowel adhesions, better wound healing, decreased requirement of analgesia. ${ }^{18}$ Regarding short term results we had very less $(8 \%)$ complications compared to $15 \%$ reported in recent literature (15\%). ${ }^{19}$ As a possible cause of bowel dysfunction postoperatively (intestinal neuronal dysplasia, hypoganglionosis etc) we found intestinal neuronal dysplasia in one. ${ }^{11,19}$

There are various systematic reviews and comparative study on heterogenous sample for laparoscopic, laparotomy Duhamel or transanal pull through but limited follow up data available on longterm functional results. As with any other laparoscopic procedure, 
adequate surgical experience is of utmost importance for the success of procedure and outcomes. ${ }^{21-23}$ Similarly observational design of our study may be have biased results. Despite our difficulties at the beginning of our experience with LADPT, we think that this technique is quick, effective and has excellent early results.

\section{Acknowledgments}

None.

\section{Conflicts of interest}

Ashish Wakhlu, Sunita Singh, Niraj srivastava nd Intezar ahmed declare that they have no conflict of interest.

\section{References}

1. Georgeson KE, Fuenfer MM, Hardin WD. Primary laparoscopic pullthrough for Hirschsprung's disease in infants and children. $J$ Pediatr Surg. 1995;30(7):1017-1022.

2. Saleh W, Rasheed K, Mohaidly MA, et al. Management of Hirschsprung's disease:a comparison of Soave's and Duhamel's pull-through methods. Pediatr Surg Int. 2004;20(8):590-593.

3. Arts E, Botden SMBI, Lacher M, Sloots P, Stanton MP, et al Duhamel versus transanal endorectal pull through (TERPT) for the surgical treatment of Hirschsprung's disease. Tech Coloproctol. 2016;20(10):677-682.

4. Georgeson KE, Robertson DJ. Laparoscopic-assisted approaches for the definitive surgery for Hirschsprung's disease. Semin Pediatr Surg. 2004;13(4):256-262.

5. Travassos DV, Bax NMA, Van der DC Zee. Duhamel procedure:a comparative retrospective study between an open and a laparoscopic technique. Surg Endosc. 2007;21(12):2163-2165.

6. Curran TJ, Raffensperger JG. Laparoscopic Swenson pull through:a comparison with the open procedure. J Pediatr Surg. 1996;31(8):11551157.

7. Lagausie P, Berrebi D, Geib G, et al. Laparoscopic Duhamel procedure Management of 30 cases. Surg Endosc. 1999;13(10):972-974.

8. Smith BM, Steiner RB, Lobe TE. Laparoscopic Duhamel pull through procedure for Hirschsprung's disease in childhood. $J$ Laparoendosc Surg. 1994;4(4):273-276.

9. De la Torre-mondragon L, Ortega-Salgado JA. Transanal endorectal pull-through for Hirschsprung's disease. J Pediatr Surg. 1998;33(8):1283-1286.

10. Muensterer OJ, Chong A, Hansen EN, et al. Single-Incision Laparoscopic Endorectal Pull-Through (SILEP) for Hirschsprung Disease. J Gastrointest Surg. 2010;14(12):1950-1954.
11. Kim AC, Langer JC, Pastor AC, et al. Endorectal pull-through for Hirschsprung's disease-a multicenter, long-term comparison of results: transanal vs transabdominal approach. J Pediatr Surg. 2010;45(6):12131220.

12. Bonnard A, de Lagausie P, Leclair MD, et al. Definitive treatment of extended Hirschsprung's disease or total colonic form Laparoscopic pull-through technique Surg Endosc. 2001;15(11):1301-1304.

13. Langer JC, Fitzgerald PG, Winthrop AL, et al. One-stage versus two stage Soave pull-through for Hirschsprung's disease in the first year of life. J Pediatr Surg. 1996;31(1):33-36.

14. Kim CY, Park LG, Park KW, et al. Adult Hirschsprung's disease Results of the Duhamel's procedure. Int J Colorect Dis. 1995;10(3):156-160.

15. Hedlund H, Hagberg S, Rubensson A, et al. Use of a circular stapler for the surgical treatment of Hirschsprung's disease. Pediatr Surg Int. 1989;4(4):291-294.

16. Saing H, Lee JMH, Tam PKH. The use of the disposable intraluminal stapler in the definitive management of Hirschsprung's disease. Dis Colon Rectum. 1986;29(3):211-213.

17. Hedlund H. Restorative proctocolectomy with J-pouch ileoanal stapled anastomosis and preserved anal canal in children. Pediatr Surg Int. 1994;9(1-2):59-60.

18. Duron JJ, Silva NJ, du Montcel ST, et al. Adhesive postoperative small bowel obstruction:incidence and risk factors of recurrence after surgical treatment:a multicenter prospective study. Ann Surg. 2006;244(5):750757.

19. Osterheld MC, Meagher-Villemure K, Ciola AM, et al. Hirschsprung's disease:the "Swiss roll" technique revisited. Pediatr Surg Inter. 2009;25(7):573-578.

20. Gobran TA, Ezzat A, Hassan ME, et al. Redo transanal endorectal pullthrough:a preliminary study. Pediatr Surg Int. 2007;23(2):189-193.

21. Giuliani S, Betalli P, Narciso A, et al. Outcome comparison among laparoscopic Duhamel, laparotomic Duhamel, and transanal endorectal pull-through:a single-center, 18-year experience. $J$ Laparoendosc $A d v$ Surg Tech A. 2011;21(9):859-863.

22. Laughlin DM, Friedmacher F, Puri P. Total colonic aganglionosis:a systematic review and meta-analysis of long-term clinical outcome. Pediatr Surg Int. 2012;28(8):773-779.

23. Gosemann JH, Friedmacher F, Ure B. Open versus transanal pullthrough for Hirschsprung disease:a systematic review of long-term outcome. Eur J Pediatr Surg. 2013;23(2):94-102. 